\title{
Exploring Orderliness by Self-Organization
}

\author{
Cheng-Yuan Liou and Wen-Pin Tai \\ Department of Computer Science and Information Engineering, \\ National Taiwan University, Taipei, Taiwan, 10764, R.O.C. \\ correspond. addr., Cheng-Yuan Liou, National Taiwan University, \\ Taiwan, 10764, R.O.C. Tel: 88623630231 ext.3228, \\ Fax: 8862 3628167, e-mail: cyliou@csie.ntu.edu.tw
}

\begin{abstract}
In an attempt to explore the orderliness by self-organization among populations, we find the idea can be applied to solve the optimization problem. We illustrate the idea and method with many varieties of the travelling salesman problem (TSP) examples. In our method a simple geometric order of the neurons is implicitly designed for the problem. The loci of the cities are represented by proper templates. The solution of the round tour will be displayed by self-organization of the templates on these ordered neurons. We test the efficiency of the proposed method by comparing the statistics of simulation results with an another potential methods [2]. Our method can be extended to both multi-dimensional distributed cities and non-straight route lengths between cities.
\end{abstract}

\section{Introduction}

We will focus our work on solving and reviewing the travelling salesman problem (TSP) and its related worked. The self-organization method has been widely applied to many applications [1]. A variety of the method has been devised to solve the TSP with many advantages [2]: Hopfield and Tank [4] have introduced a popular and attractive method to solve the TSP based on a popular neural network model. Their method has induced many valuable improved versions. We will introduce a new approach to solve the TSP using the canonical self-organization algorithm given by Kohonen in [1] following our idea. We start with a properly and intuitively designed geometric distribution of the neurons. The geometric distribution of the neurons has the order which reflects the needed solution implicitly. For the TSP, the number of neurons is the same as the number of cities and the neurons are located on an uniform ring. The loci of the cities are used as templates. The self-organization of these templates on the ring of neurons will converge to a proper solution for the TSP. In other words, we formulate the optimization problem into a standard template self-organization problem. This contrasts sharply the traditional pattern recognition approach which formulates the pattern recognition problem into an optimization problem. The implicit orderliness of the distribution of the neurons is the key to the proposed method. We will introduce the detailed method in next section. We will test the efficiency of the proposed method by comparing with a potential method [2] in a later section.

\section{Method}

Since the evolution of self-organization will generate a topology-preserving map which keeps the shortest relationship among neighbors and keeps the complex relationship among all neighbors, this initiates an attempt to fit it for optimization problem. The complex relationship can be reduced by a simple geometric distribution of the neurons which implicitly configures the solution. For the TSP cases, the neurons are located on an uniform circular ring. The loci of the cities compose the set of templates. Then we can applied the standard self-organization algorithm for the set of templates on the neuron ring, see figure 1. Each neuron on the ring has the same number of synapses as the number of dimensions of the template. The total number of the neurons is the same as the total number of cities. When convergence is reached, we expect each neuron represents a different city and the sequence of the representation on the neuron ring displays a proper solution for the given TSP. The loci of the cities compose the set of training templates which can be given as a set of plane coordinates 
$\left\{\left[x_{i}, y_{i}\right]^{T} \mid \mathrm{i}=1,2, \ldots, \mathrm{n}.\right\}$ of the $\mathrm{n}$ cities, or relative distances between cities $\left\{\left[d_{i 1}, d_{i 2}, \ldots, d_{i k}, \ldots, d_{i n}\right]^{T} \mid\right.$ $\mathrm{i}=1, \ldots, \mathrm{n}$.$\} , or actual road distance between cities \left\{\left[d_{i 1}, d_{i 2}, \ldots, d_{i n}\right]^{T} \mid \mathrm{i}=1, \ldots, \mathrm{n}\right.$. $\}$. Where $\left[x_{i}, y_{i}\right]$ is the plane coordinate of the $i^{\text {th }}$ city, $d_{i k}$ is the relative straight or non-straight distance between city $\mathrm{i}$ and $\mathrm{k}$. The synapses of each neuron compose the representing pattern, $\left\{\mathrm{n}_{i}=\left[w_{i 1}, w_{i 2}, \ldots, w_{i n}\right]^{T} \mid\right.$ $\mathrm{i}=1, \ldots, \mathrm{n}$.$\} , of that neuron. We use w_{j k}$ to denote the $k^{\text {th }}$ synapse of the $j^{\text {th }}$ neuron. $\underline{n}_{i}$ denotes the representing pattern of the $i^{\text {th }}$ neuron.

The process for each learning cycle consists of two steps: the first step involves finding the bestmatching neuron for each training template or citys locus under the rule that the closest neuron is selected to each city. Because the cities are processed in a random order, it may happen that a selected neuron has been already assigned to a previously processed city. This problem can be solved by re-arranging the latter city to match the neighbouring neuron of this best-match one for preserving the neighborhood relationships of the input templates in the feature map. This arrangement will effect the circularly linked map to achieve the desired solution of the shortest path.

After the injective mapping from cities to neurons has been built, the second step is to update the synapses of every neuron and its surrounding neighbors toward the neurons corresponding city template. The area of the chosen neighborhood, $N_{c}(\mathrm{t})$, will shrink as the training proceeds. Because of the one-to-one correspondence from cities to neurons in each cycle, the training process will lend every neuron win the competition and have their weights adjusted with equal probability, therefore each updating will be efficient for convergence [3].

In the self-organization algorithm, there are two parameters, the range of topological neighborhood $N_{c}(\mathrm{t})$ and the gain parameter $\alpha(\mathrm{t})$. Both are designed as linearly decreasing functions of time in all our simulations, and are initialized with proper values. To clearly illustrate the proposed method, we give the algorithm for the n-city TSP in the following context:

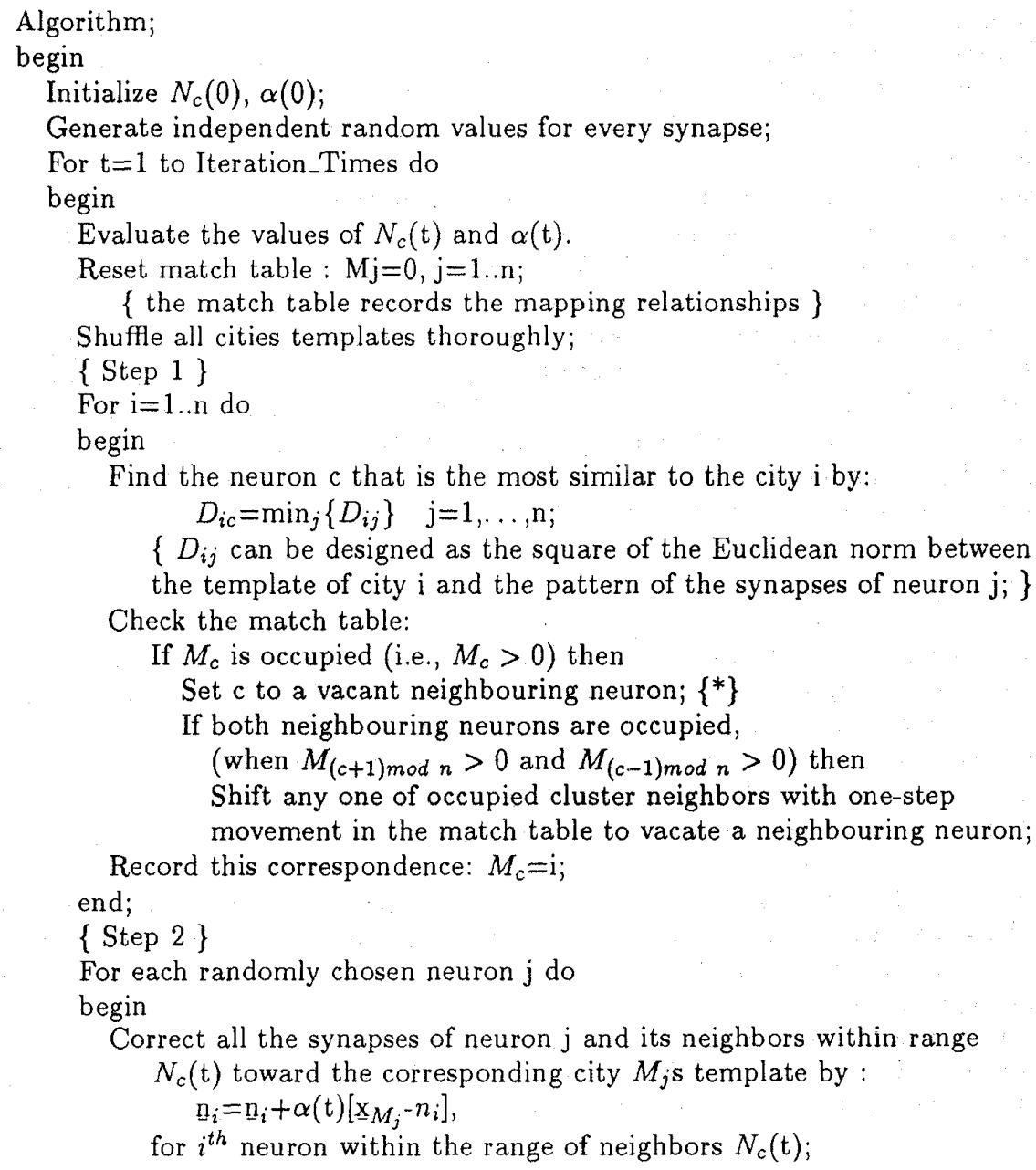




$$
\begin{aligned}
& \text { where } \mathrm{x}_{M_{j}} \text { is the template (or locus) of the city } M_{j} \text {. Note that } \\
& \underline{n}_{i}=\left[w_{i x}, w_{i y}\right]^{T} \text { and } x_{i}=\left[x_{i}, y_{i}\right]^{T} \text { are used in plane coordinate template case, } \\
& \underline{\underline{n}}_{i}=\left[w_{i 1}, w_{i 2}, . . w_{i k}, . . w_{i n}\right]^{T} \text { and } \underline{x}_{i}=\left[d_{i 1}, d_{i 2}, . . d_{i k}, . . d_{i n}\right]^{T} \text { are used in relative } \\
& \text { end; } \\
& \text { distance template case. } \\
& \text { end; } \\
& \text { end. }
\end{aligned}
$$

In the above algorithm we divide one learning cycle into a matching step and an updating step for all neurons. We also develop an one-to-one matching correspondence in each cycle for achieving conscious efficiency [3] indirectly.

\section{Simulation Results}

In a series of simulations, the performance of this algorithm is investigated using several varied cases. In order to speed up the training, we adopt a linked list to implement the match table used in program. As to the strategies to locate the latter cities* (see the algorithm) in properly neighbouring places on the circular ring of neurons, existing techniques can be employed with similar performance. In our simulations we set the initial value for the parameter $\alpha(0)$ to 0.5 , and the initial value for $N_{c}(0)$ to $60 \%$ of all neurons. The results of simulations are obtained when $\alpha$ reaches zero.

Figure 2(a) shows the simulation results with two different methods in solving the TSP: the method proposed by [2], and our method. A tour length distribution graph of the two methods after 2000 executions for a 30 city TSP is shown in this figure. The 30 cities are generated randomly. And we use the city's coordinates $\left[x_{i}, y_{i}\right]$ as templates. The best solution of our method for the above case is shown in figure $2(b)$.

Simulation results for large number of cities are also included. A tour length distribution graph are displayed in figure 3 for a 532-city problem [5], of which the length of the shortest path is known. Figure 4 shows a satisfactory solution in one execution for 1000 cities by our method, where there is no route intersection.

For multi-dimensional distributed cities, we use the following simulation as a 3-dimension example. Simulation result for 10 three-dimensional randomly distributed cities is shown in figure 5 . See table I for the locations of the 10 cities. The 3 -dimension coordinates $\left[x_{i}, y_{i}, z_{i}\right]$ are used as training templates. The representing pattern of the synapses of each neuron is $\left[w_{i x}, w_{i y}, w_{i z}\right]$ accordingly.

Furthermore, our algorithm can be also extended to problems in which the route length from one city to another is not a straight line but a distorted route. The actual route lengths between cities can also be organized in a matrix, each row of the matrix represents a template. Thus $n$ templates can be derived for the $\mathrm{n}$ cities. The training processes are still the same as the original algorithm. From many results of simulations, our method can be applied to solve the TSP with non-straight distances between cities practically and efficiently.

\section{Conclusion}

In this work we formulate the TSP in a new self-organization approach. Many various simulations support our approach for optimization problems. The origin of the idea is to find the mechanism for orderliness among populations. Each one can be represented by many dimensions. The evolution of self-organization among the population will reorganize themselves into order. This order is important for many civil designs. The idea of our approach is totally different from the conventional techniques for optimization problems. And this approach offers a new practical way to solve the optimization problems.

Our approach can be easily extended to both three-dimension distributed cities and non-straight route length between cities. This will fit many practical problems. As far as we know our method is the only practical and efficient method for solving the general TSP with multidimensional nonstraight route length between cities.

Our method contrasts with the conventional pattern recognition approach which formulates the pattern recognition problem into an optimization problem. We formulate optimization problem into pattern self-organization problem. Many simulation results has verified our method. 


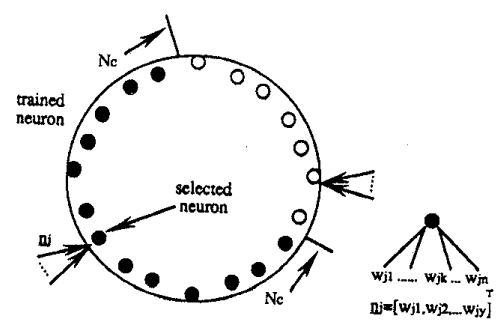

Figure 1. The neuron ring. Each neuron on the ring has the same number of synapses as the number of dimensions of the template.

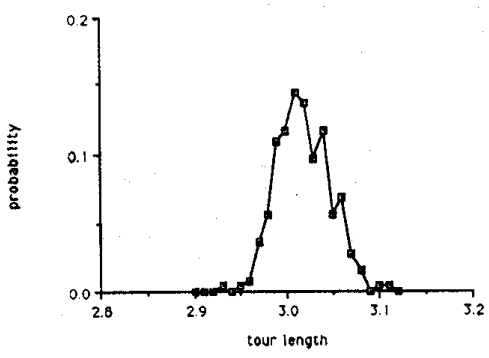

Figure 3. The statistics for a TSP with 532 cities [5], of which the length of the shortest path is known. The length of the shortest path is 2.7686 . The distribution graph of the tour length by our algorithm is shown in this graph.
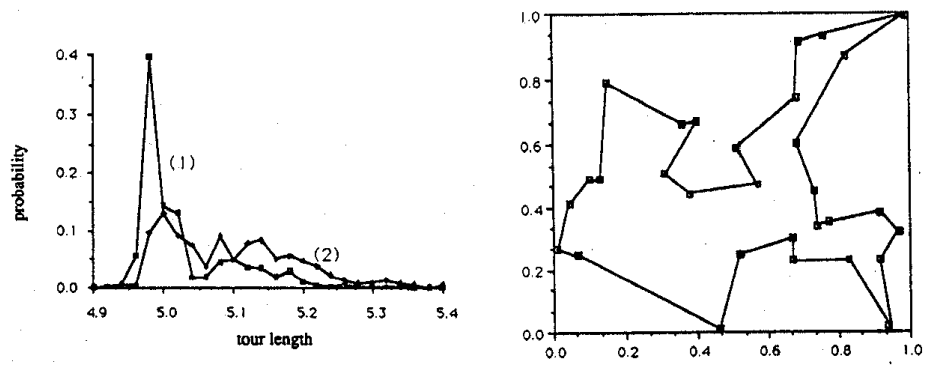

(a) The distribution graph of the tour length by the two methods after 2000 executions: line (1) represents the results by the method [2] ( $\alpha=0.02)$, and line (2) represents the results by our method with 400 iterations.

(b) The mar, of the 30 cities and the best solution found by vur method.

Figure 2. The simulation results for the 30 city case.

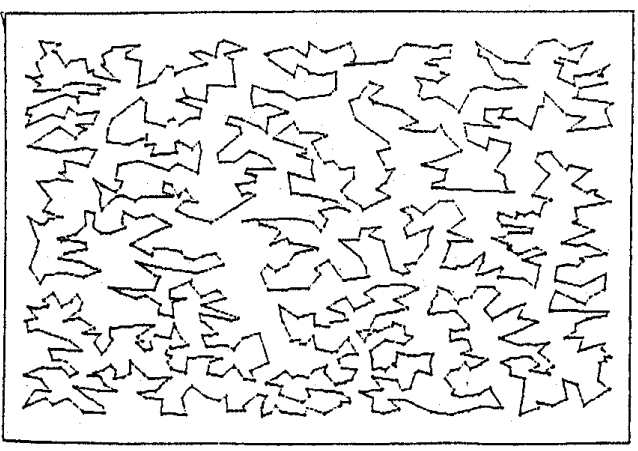

Figure 4. A satisfactory tour. (no route intersection) for a 1000 city case. It took 110 minutes totally for a $\mathrm{PC} 486 \cdot$ with 1000 iteration times.

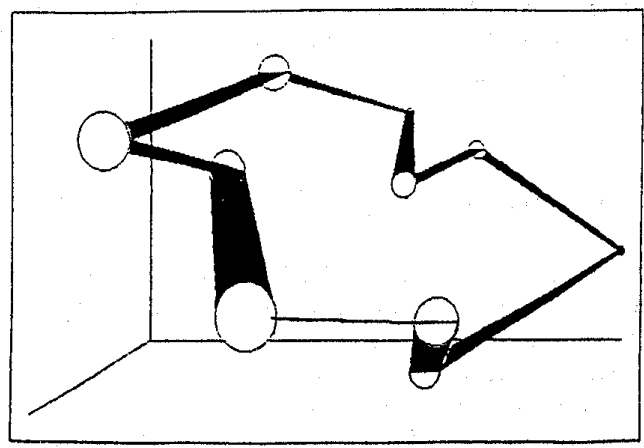

Figure 5. Simulation results for 10 three dimensionally distributed city case in table I. The 3 dimensional graph of the best solution for this case is shown.

\section{References}

[1] T. Kohonen, Self-Organization and Associative Memory, Springer-Verlag, Berlin, 1988.

[2] B. Angeniol, G. de La Croix Vaubois, and J-Y. Le Texier, "Self-Organizing Feature Maps and the Travelling Salesman Problem", Neural Networks, Vol. 1, 1988, pp. 289-293.

[3] D. DeSieno, "Adding a Conscience to Competitive Learning", Proc. 1988 IEEE ICNN, Vol. I, San Diego, California, July 1988, pp. 117-124.

[4] J. J. Hopfield and D.W. Tank, "Neural Computation of Decisions in Optimization Problem", Biological Cybernetics, 52, 1985, pp. 141-152.

[5] M. Padberg and G.Rinaldi, "Optimization of A 532-City Symmetric Traveling Salesman Problem by Branch And Cut", Operations Research Letters, Vol. 6, No. 1, 1987, pp. 1-7. 